\title{
Contributing Reviewers in 2015
}

J Pediatr Genet 2016;5:i.

Journal of Pediatric Genetics wishes to recognize those who contributed as an expert peer reviewer of submitted scientific papers in 2015.

Thank you for your contributions to Journal of Pediatric Genetics in 2015.

Ahmed Abdelhalim
Patrizia Agretti
Mohammad Al-Haggar
Kevin M. Antshel
Maarten Arends
Linlea Armstrong
Lokman Aslan
Said Azoury
Jong Bhak
Erwin Brosens
Anna Capozzi
Ingele Casteels
Xu Chenming
Brian Hon-Yin Chung
Peter Conner
Seth Joel Corey
Goran Cuturilo
Angie Dawson
Johnny Deladoëy

Ahmed Abdelhalim

Mohammad Al-Haggar

Kevin M. Antshe

Linlea Armstrong

Lokman Aslan

Said Azoury

Jong Bhak

Erwin Brosens

Anna Capozzi

Ingele Casteels

Xu Chenming

Brian Hon-Yin Chung

Goran Cuturilo

Johnny Deladoëy

Maude Le Gall
Deike Hesse
Hans L.J. Hoeve
David Hsieh
Naoko Ishihara
Janez Jazbec
Hiroshi Kanno
Claudia Kappen
Yskert von Kodolitsch
Alfried Kohlschütter
Dawn Laney
Thomas Liehr
Ahmad M. Mansour
Bruno Marino
Guiseppe Merla
Takayuki Morisaki
Kasiani Myers
Julián Nevado
Ashley Parrott

Millan Patel

Claudio Pignata

Helton E. Ramos

Aino Rantala

Ilena Rubio

Osamu Sakamoto

Mari Satoh

Kim Summers

Toshihiro Tajima

Dick Tibboel

Albena Todorova

Suni Trivedi

Lucie M. Turcotte

Judith M.A. Verhagen

Steven G. Waguespack

Michiko Watanabe

Varina Wolf 